\title{
Approaching the Next Revolution? Evolutionary Integration of Neural and Immune Pathogen Sensing and Response
}

\author{
Kevin J. Tracey \\ Feinstein Institute for Medical Research, Manhasset, New York 11030 \\ Correspondence: kjtracey@nshs.edu
}

Mammalian immunity evolved by the process of natural selection that produced differential survival and reproduction advantages through combinations of hereditary traits underlying the response to pathogens. Primitive animals sense the presence of microbial pathogens through recognition of pathogen-derived molecules in their rudimentary immune and nervous systems. No molecular biological mechanism assigns primacy of pathogen sensing mechanisms to immune cells over neurons. Rather, in animals as diverse as Caenorhabditis elegans to mammals, neural reflexes are activated by the presence of pathogens and transduce neural mechanisms that control the development of immunity. A coming revolution in immunological thinking will require immunologists to incorporate neural circuits into understanding pathogen signal transduction, and the molecular mechanisms of learning, that culminate in immunity.

$\mathrm{O}_{\mathrm{r}}^{\mathrm{n}}$ considering memory, one finds an ironic perspective, tainted by shadows of two major scientific fields that, historically at least, did not collaborate. Immunological memory, mediated by lymphocytes, and neurological memory, mediated by neurons, evolved over millions of years in response to environmental changes. Closer inspection within both fields reveals key features of common origin between neural and immune information collection and retrieval. Major evolutionary advantages arose at points of intersection of these systems, manifested as beneficial physiological responses to environmental stimuli during infection, injury, and metabolic stress. In 1989, Charles Janeway proposed that the first revolution in immuno- logical thinking led to the domination of the humoral theory of immunity, and that an approaching second revolution would integrate innate and adaptive immunity by understanding the role of pathogen-associated molecular pattern receptors (Janeway 1989). Today, I believe that the groundwork has been laid for a third revolution in immunological thinking that will integrate the role of neurological feedback circuits into innate and adaptive immunity, including the role of molecular mechanisms through which neurons sense microbes and regulate the output of hematopoietic-derived immune cells. I also suggest that costimulation of neural reflex circuits by microbial products plays a major role in the immune response to

Editors: Ruslan M. Medzhitov

Additional Perspectives on Innate Immunity and Inflammation available at www.cshperspectives.org

Copyright (C) 2015 Cold Spring Harbor Laboratory Press; all rights reserved; doi: 10.1101/cshperspect.a016360 Cite this article as Cold Spring Harb Perspect Biol 2015;7:a016360 
K.J. Tracey

infection, and to the subsequent development of immunity (Fig. 1). If correct, this idea could revolutionize our thinking about immunity and take us beyond innate and adaptive immunity to an integrated view of neurological and immunological recognition and learning.

\section{EVOLUTIONARILY ANCIENT SENSING MECHANISMS IN NEUROSCIENCE AND IMMUNOLOGY}

It begins with sensing a change in the environment. Consider the evolutionarily primitive animal, Caenorhabditis elegans, which harbors rudimentary immune and nervous systems. Moving and feeding within a bacterial lawn, it uses neural and immune sensory mechanisms to detect the presence of pathogenic microbes and toxins (Pradel et al. 2007). On encountering specific molecules, sensory neurons transduce the chemical environmental information into action potentials, which initiate neural responses that modify the animal's behavior and motion. The presence of pathogens also activates its innate immune cells, which incite defensive reactions, including enhanced MAP kinase and XBP-1 signaling, accumulation of unfolded proteins in endoplasmic reticulum, and other cellular and metabolic responses (Styer et al. 2008; Aballay 2013). Sensory neural signals initiate primitive response circuits linked to corresponding motor neural signals that determine behavioral and physiological responses. Thus, the worm's neural and innate immune sensory systems independently detect the presence of the pathogens in the environment.

Primitive innate immune-like cells in C. elegans, which predate the evolution of competent Toll-like receptor and NF- $\mathrm{\kappa B}$ signal transduction mechanisms, are also activated by the presence of pathogens or injury (Aballay 2013). Gene expression patterns in the innate immune system, and animal behavior mediated by the nervous system, are altered in a pathogen species-dependent manner. The result of these responses is that the animal can defend itself against certain pathogen encounters, although not all. The innate neural and immunological

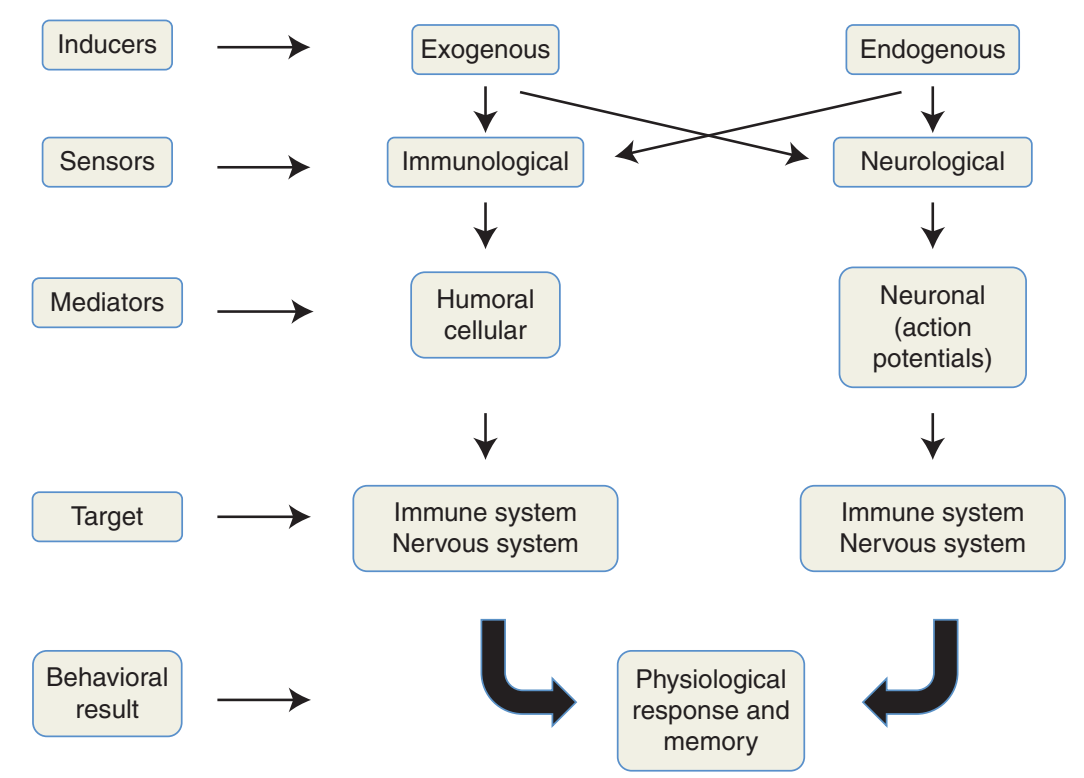

Figure 1. Pathogens or products of cellular inflammation and injury costimulate immune cells and neurons during the earliest stages of infection. Neural input activates reflex circuits, which modulate the nervous system and the immune system. Hematopoietic-derived cellular responses produce humoral and cellular signals that influence immune cells and neurons. These signal transduction pathways culminate in mediating the behavior of the animal and in initiating learning. 
sensing events are both operative at the earliest stage of the encounter, but the speed of neural transmission offers a key advantage to coordinating the animal's specific responses.

There are 302 neurons in C. elegans, and the interconnections and response characteristics of these have been elucidated. A characteristic feature of the worm's response to infection is that one family of neural circuits, activated when sensory neurons detect the presence of pathogenic pseudomonas, transmits neurotransmitter-dependent signals to mediate pathogen avoidance behaviors, and to suppress the unfolded protein response in innate immune cells (Sun et al. 2011). In light of today's advanced knowledge, and our detailed molecular mechanistic understanding about inhibitory reflex control of innate immune responses in mammals, perhaps the perspective becomes clearer. The regulatory intersection between the nervous and innate immune systems in C. elegans, which has been retained in today's mammals, originated from evolutionary pressure to optimize physiological homeostasis, and survival.

Beyond either field, there is little need to account for separateness in functional mechanisms that operate in a parallel time frame, and also intersect to control the magnitude of host defense and response. But immunology and neuroscience have only recently embraced this theory, so there is value in reviewing the concept from the perspectives originating within each. From an historic perspective in immunology, the principal focus has been to understand the development and maintenance of immunity, and to provide a complete molecular mechanistic basis for how lymphocytes acquire and retain the information necessary to remember the nature of infectious threats. This has been highly successful for establishing the principles to explain the diversity and repertoire of long-term humoral and cellular immune memory that results from antigen processing, presentation, and clonal development of B and T cells. A detailed molecular understanding for the basis of innate immunity was also produced. When likened to reflex or instinctual behavior, pathogen recognition by innate immune cells is encoded in the genome, enables a relatively rapid and consis- tent response to pathogen-associated and damaged-associated molecular patterns, and is highly conserved. These mechanisms of immunity have been exceedingly well described within a knowledge sphere circumscribed by cells of hematopoietic origin, operating in response to pathogens, absent control from other systems. It is axiomatic within immunological thinking that the earliest stages of sensing pathogens occurs through activation of innate immune pattern-recognition receptor signals, and that later sensing occurs through adaptive immune responses.

In an historic time line parallel with these unfolding events, the principle focus of neuroscience has been to understand the functions of the brain and nervous system. This established neurons as the principal cells controlling information, learning, and memory; the chemical basis of neurotransmission; and the principles of synaptic neural communication underlying neuronal signaling. In the mid-20th century, Sherrington famously recognized that the simple reflex, comprised of a sensory arc, interneurons, and a motor arc, is the fundamental unit for information processing on which the entire nervous system evolved (Sherrington 1906). From C. elegans and to modern mammals, sensory neurons respond to chemical, mechanical, or temperature changes in the environment. This information is transduced via ion channels and gradient potentials that generate action potentials that are relayed to other neurons. During pathogen threat, or in response to other environmental changes, information processing in the nervous system begins with sensory input transmitted in the afferent arc of a reflex circuit.

Sensory neural signals in mammals are routed through nuclei residing within the central nervous system, primarily in the brain stem. These nuclei produce the output that regulates organ function by transmission through the motor arc to complete the reflex circuit. The simple reflex unit is the basic building block that evolutionary pressure used to integrate and assemble the mammalian nervous system as the regulatory and coordinating system of host responses to infection, injury, and other threats. Nuclei that provide the relay stations 
K.J. Tracey

for regulatory reflexes are housed in the evolutionarily ancient regions of the mammalian brain stem. These brain stem nuclei control the homeostatic set points of all basic physiological functions associated with health, and the response to environmental change.

Individual neural reflex circuits are stimulated in response to specific sensory inputs arising within the body organs. The reflexively controlled release of neurotransmitters from the motor arc controls the output of each organ. Ultimately, the physiological, or involuntary, behavior of the host is the result of the sum total action of all reflexes connecting its organs to its brain stem. These involuntary reflexes function with limited or no input from the cerebral cortex. They operate largely beneath conscious control. Primitive animals, like C. elegans, which (presumably) lack the cerebral faculties of consciousness and self-awareness, also show reflex behavior in response to changes in pressure, temperature, the chemical composition of the environment, and the presence of pathogens.

In considering the role of regulatory feedback on the molecular mechanisms underlying immunity, it bears special note here that there is a modern tendency to center discussions of mammalian neural information processing on the cerebral cortex. Termed "cortical conceit," one may view the sophisticated human cerebral encephalon as an undoubted source of inestimable pride, a grand evolutionary accomplishment (Dubos 1998). But the advanced cerebral cortex found in mammals is a relatively new addition to biology, one that is largely dispensable for understanding basic mechanisms of physiological and immunological homeostasis. Surgically or genetically decorticated mammals can well maintain physiological and immunological set points within a healthy range, as long as the brain stem nuclei and corresponding reflex circuits are functional. Basic reflex circuits, having developed in an evolutionary time, maintain physiological responses during infection and injury without requiring input from the cortex to operate competently. Signals descending from the higher brain can influence the functional output of the reflex nuclei, but these psychological implications need not be considered here to understand the basic physiological principles governing homeostasis. So, in defining rules that govern how the nervous system modulates the immune system to establish immunological homeostasis, we can dispense with the need to consider individual acts of conscious will, or choices made. The system operates in the background of conscious existence, driven by sensory inputs that elicit reflex responses.

These reflex circuits originating in the evolutionary precursors of the modern mammalian brain stem nuclei conferred the ability to modulate cardiac and pulmonary function in response to infection, and countless other protective and beneficial organ responses. Animals expressing such competent beneficial reflexes gained a preservation and reproductive advantage over breeding populations lacking it. In time, the accumulation of individual simple reflexes endowed evolving species with optimal flexibility necessary to use effective defensive responses and maintain homeostasis in response to a range of changes in the internal and external milieu. The identity and function of these regulatory reflexes in mammals was revealed in classic physiological studies of the anatomically accessible body systems. Consider the cardiovascular system, which is beautifully presented in the thorax when the sternum is divided, to behold the beating heart.

With each cardiac contraction it is possible to observe, measure, and record numerous experimental end points reflecting the intricate physiological working of the reflexively controlled heart. Cutting the vagus nerve to the heart produces an increase in heart rate, indicating that heart rate is tonically suppressed by descending neural signals from the brain stem. This descending inhibitory signal is transmitted in the motor arc of a reflex initiated when an increase in heart rate is sensed by afferent baroreceptor neurons. Action potentials arrive within brain stem nuclei, and from there, neural signals exit the brain stem to be transmitted back to the heart to decrease heart rate relative to a predetermined set point. Other reflexes, activated when heart rate decelerates below its set point, culminate in signaling via other mo- 
Integration of Neural and Immune Pathogen Sensing

tor arcs that increase heart rate. From observations of this sort, physiologists and neuroscientists built detailed models and developed rules of feedback control based on individual units of reflex action to explain how cardiovascular homeostasis is maintained during a range of changes in the internal and external milieu. Similar rules have been developed and applied to other organ systems. The regulatory neural systems that reflexively control physiological homeostasis operate in real time, are highly sensitive to changes in external and internal environment, and ultimately enable organ systems to function within a narrow range of metabolic, thermal, nutrient, and behavioral parameters that is compatible with cellular and animal health.

The vagus nerve is a principal nerve in mammals that transmits afferent, sensory information from the organs to the brain stem, and it is a mechanism for informing the nervous system about the status of the internal milieu. From the visceral organs, it travels a meandering course across the abdomen, thorax, and neck, entering the brain stem, where its sensory nerve endings synapse on, and relay information to, neurons in the nucleus tractus solatarius. Interneurons arising there relay signals to the dorsal motor nucleus of the vagus nerve, representing a major cholinergic outflow tract that returns to the peripheral organs. The motor arcs stimulated by vagus sensory afferent neurons return to the organs via efferent neurons that travel along two major routes: the sympathetic chain, and the vagus nerve itself. At the nerve terminus, motor neurons from these two major outflow paths release acetylcholine, norepinephrine, vasoactive peptide, and other neuropeptides that bind to cognate receptors in the target organ's epithelial and parenchymal cells, to modulate cellular metabolism, function, and secretory capacity. Studies of the cardiopulmonary, gastrointestinal, renal, hepatic, and endocrine systems established the anatomic and neurophysiological principles underlying reflex control of physiological homeostasis. Until quite recently, however, the existence of regulatory reflexes exerting control over the immune system had evaded description. Immunological thinking had not viewed immunity as a reflexively regulated response.

\section{REFLEX REGULATION OF INNATE IMMUNITY}

This began to change following the discovery that cytokines produced in response to infection and injury are necessary and sufficient mediators in disease pathogenesis. This provided experimental tools and strategies that enabled the illumination of neural circuits that regulate innate immunity. Termed "the cytokine theory of disease," cytokines produced in response to infection and injury mediate cellular, metabolic, and pathological effects underlying the major physiological and clinical manifestations of illness (Tracey 2007). Restated, disease occurs when there is a loss of immunological homeostasis producing an unbalanced or excessive cytokine response. In such cases, inhibiting or modulating the cytokine response to restore immunological homeostasis can prevent or reverse disease, and it should be stressed that the cytokine theory does not state that all cytokines are "bad." By analogy, the germ theory does not state that all microbes are pathogenic. Rather, the cytokine theory indicates that in some instances of cytokine imbalance or excess, treatment of diseases can be specifically accomplished with targeted therapeutics.

The cytokine theory of disease was validated in mammals, including humans, within a few years after early direct evidence became available that tumor necrosis factor (TNF) is a necessary and sufficient mediator of acute septic shock during infection (Tracey et al. 1986, 1987). The first principles for administering anti-TNF monoclonal antibodies as therapy were established in baboons infected with Escherichia coli (Tracey et al. 1987). Treated animals survived despite the presence of replicating bacteria in their bloodstream, proving that the disease (septic shock) required the activity of TNF. Disease was attributable to the activity of the cytokine, regardless of the status of the pathogen (Tracey et al. 1987). Today it is common to witness this principle being applied to patients receiving monoclonal anti-TNF for the treat- 
K.J. Tracey

ment of rheumatoid arthritis, inflammatory bowel disease, and other conditions. From a practical point of view, the earliest underlying or inciting etiological agent can be dissociated from the disease. The physician standing at the patient's bedside is witness to signs and symptoms of inflammation, organ damage, and toxicity mediated by the direct action of cytokines. In many, if not most, diseases, excessive cytokine activity can be considered as a failure of immunological homeostasis.

It was from this perspective, in the 1990s, that my colleagues and I began to directly address the possibility that reflex neural circuits had a critical role in regulating cytokine release and maintaining immunological homeostasis. We focused on the TNF response in animal models of infection and injury. The choice of TNF as the target of these studies was important, because it represents an early-stage response, rapidly induced in response to pathogenic stimulation. The measurable TNF response falls within a time window peaking 90 min after exposure to endotoxin, quite compatible with making observations that could be interpreted in the context of experimental manipulation of neural circuits. To determine whether neural signals suppress TNF release from tissue macrophages, we applied stimulating electrodes to the vagus nerve where it traversed the neck on its descending course to the reticuloendothelial system (Borovikova et al. 2000). Electrical stimulation of the vagus nerve significantly inhibited TNF release in red pulp and marginal zone macrophages in spleen, suggesting that descending signals in the motor arc of an "inflammatory reflex" participate in regulating the innate immune response and maintaining immunological, or "cytokine homeostasis" (Tracey 2002; Rosas-Ballina et al. 2008).

When the vagus nerve was divided before exposing animals to endotoxin, we observed that TNF production by splenic macrophages was enhanced (Borovikova et al. 2000). By analogy to the tonic inhibitory influence of vagus nerve signaling, which inhibits heart rate, this fact indicated that constitutively active or tonic vagus nerve signals slow or inhibit the innate immune response to endotoxin. By following the track of the vagus nerve from the brain stem, down the neck, across the thorax, and into the abdomen, and either dividing the nerve in some cases, or electrically stimulating it along its course in others, we discovered that the point of convergence of the vagus nerve signals to regulate serum TNF during endotoxemia is in the spleen (Huston et al. 2006; Rosas-Ballina et al. 2008). Electrical signals applied to the cervical vagus nerve descend to the celiac ganglion, which is the origin of motor neurons that innervate the spleen. Vagus nerve and splenic nerve action potentials inhibit the release of TNF in red pulp and marginal zone macrophages, which together account for $>90 \%$ of the peak serum TNF levels following the acute onset of endotoxemia.

Three implications from these findings are immediately obvious. First, the TNF producing innate immune response to endotoxin is regulated by neural signals. The constitutive inhibitory activity of neural circuits establishes a set point for the innate immune response to endotoxin. Second, the immune system is anatomically and functionally innervated. Although extensive prior anatomic evidence indicated that all of the major organs of the reticuloendothelial system receive neural connections from the brain stem, the prior anatomic findings had not provided a scientific framework for experimental study of the functional operation of these neurons. Approaching this as a functional, neurophysiological property of neuronal activity to regulate the innate immune response to endotoxin opened a door to delineating molecular mechanisms. Third, it is overwhelmingly probable that the basic principles of reflex action activated by sensory input also apply to other aspects of innate immunity and adaptive immunity. The evolutionary emergence of lymphocytes, and adaptive immunity, occurred roughly in parallel with the emergence of more sophisticated neural networks arising from evolutionarily expanding neural circuits and connectivity. It is nearly impossible to imagine an evolving situation in which the environmental changes caused by infection and injury did not intersect with signals that simultaneously influence both the immune and nervous systems. 
As broadly defined, immunity is the state of acquiring (learning) and retaining (remembering) systems to confer protection against infection. On accepting the theory that neural signals, which regulate immune responses, are costimulated during the activation of innate immunity, and provide regulatory signals in the earliest stages of acquiring immune memory, then it can be strongly argued that a complete understanding of immunity requires incorporating neural mechanisms. To determine the mechanism for reflex inhibition of innate immunity, we experimentally followed the path of the neural signals arising in the vagus nerve and terminating in the spleen (Rosas-Ballina et al. 2011). Within the spleen, we observed splenic nerve endings terminating in synapse-like structures adjacent to lymphocytes. In transgenic mice, which coexpressed green fluorescent protein in cells that express choline acetyltransferase, the rate-limiting enzyme that catalyzes the biosynthesis of acetylcholine, we observed that $\mathrm{T}$ lymphocytes capable of synthesizing acetylcholine resided in close proximity to splenic nerve endings. Electrical stimulation of the vagus nerve stimulated the adrenergic splenic nerve to release norepinephrine, which in turn stimulated Chat-expressing $\mathrm{T}$ cells to release acetylcholine. Lymphocyte-derived acetylcholine is the neurally activated molecular mediator that binds to $\alpha 7$ nicotinic acetylcholine receptors expressed by red pulp and marginal zone macrophages (Wang et al. 2003). Ligand receptor interaction inhibits the release of TNF through a molecular mechanism that has been attributed to formation of a heteroprotein complex comprised of $\alpha 7$ binding to JAK-STAT that suppresses activation of the nuclear translocation of nuclear factor $\kappa \mathrm{B}$ and to stabilizing mitochrondrial membrane permeability (de Jonge et al. 2005; Lu et al. 2014). The functional importance of these $\mathrm{T}$ cells in this neural circuit is striking, because T-cell-deficient nude mice lack a working inflammatory reflex. Electrical stimulation of the vagus nerve in nude mice fails to inhibit TNF. This phenotype is reversed by passive transfer of Chat-expressing $\mathrm{T}$ cells into naïve recipient nude mice, which restores the functional inflammatory reflex in these animals
(Rosas-Ballina et al. 2011). Thus, the inflammatory reflex is defined by vagus nerve action potentials that activate the release of an inhibitory neurotransmitter, acetylcholine, from specialized lymphocytes to suppress the magnitude of the TNF response in spleen. A prototypical neural circuit prevents overexpression of TNF to establish healthy immunological homeostasis.

Our initial observations on the neural regulation of TNF release enabled us to formulate the fundamental principles for reflex control of immunity. Discrete neurons terminating in specific molecular mechanisms modulate the immune response to infection (Tracey 2009; Andersson and Tracey 2012a). Inhibitory neural control of innate immunity by acetylcholine is not restricted to controlling TNF, because acetylcholine interaction with $\alpha 7$ nicotinic acetylcholine receptors in macrophages significantly inhibits activation of the inflammasome, the protein complex required for the release of leaderless cytokines, including IL-1, HMGB1, and IL-18 (Borovikova et al. 2000; Lu et al. 2014). Expression of $\alpha 7 \mathrm{nAChR}$ is essential to the integrity of the inflammatory reflex, because $\alpha 7$ nAChR knockout mice have an impaired inflammatory reflex. Vagus nerve stimulation fails to suppress inflammasome activity in these animals; and, moreover, activation of innate immunity in the $\alpha 7 \mathrm{nAChR}$ knockout mice produces significantly higher levels of TNF and the leaderless cytokines (Wang et al. 2003; Lu et al. 2014).

\section{SENSORY STIMULATION OF NEUROLOGICAL REFLEXES IN IMMUNITY}

As is the case for all reflexes, the motor arc of the inflammatory reflex is activated in response to signals arising in the sensory arc. Beyond the evidence cited above that the interaction of C. elegans with pathogens begins by sensing signals transmitted in afferent neurons, a series of pivotal studies have provided insight into the origin and function of the sensory arc of the inflammatory reflex in mammals. Originally, Linda Watkins and her colleagues (1995) made the seminal discovery that the fever response induced by the intra-abdominal admin- 
K.J. Tracey

istration of IL-1 requires an intact and functional vagus nerve, because when they surgically divided the vagus nerve, animals maintained normothermia despite exposure to intra-abdominal IL-1. Thus, the presence of IL-1 in the abdomen, or more specifically in the hepatic circulation, is sensed by the vagus nerve, which propagates action potentials ascending to synapses in the nucleus tractus solatarius in the brain stem (Fairchild et al. 2011). Sensory vagus nerve action potentials mediated by IL- 1 have been experimentally shown using recording electrodes applied to the vagus nerve at various points along its course from the abdomen to the brain stem (Niijima 1996). Compound action potentials arising in response to intrahepatic IL1 have also been recorded in the splenic nerve, which is a pure motor nerve lacking sensory fibers (Niijima et al. 1991). This gives direct evidence that sensory vagus nerve signals activate compound action potential signals descending to the spleen via the motor arc of the inflammatory reflex.

Other inflammatory mediators have been implicated in stimulating sensory action potentials in the vagus nerve, including endotoxin, TNF, prostaglandins, substance $\mathrm{P}$, and other endogenous and exogenous products of infection and injury (Chiu et al. 2012). The sensory and motor arcs of the inflammatory reflex within the vagus nerve are positioned to anatomically and functionally respond to and modulate the host response to infection and threat in the visceral organs of the body compartment. The importance of this inhibitory neural circuit to maintaining immunological homeostasis is indicated by the findings that animals deficient in this reflex are rendered increasingly sensitive to excessive TNF release. This raises the next question: Can reflexes stimulate, rather than inhibit, innate immune responses to maintain immunological homeostasis?

As far back as 1874, it had been proposed that inflammation is mediated by action potentials transmitted in peripheral neurons (Chiu et al. 2012). This stemmed from the observations that electrical stimulation of dorsal root neurons produced vasodilation in the innervated skin. Today we understand that the in- flammatory effect of these motor neurons is attributable to neurotransmitters released into the tissue including neuropeptides, catecholamines, and substance $P$. These molecules interact with cognate receptors expressed on endothelial cells, smooth muscle cells, lymphocytes, monocytes, and macrophages that in turn mediate vasodilation, increased capillary permeability, neutrophil recruitment, and swelling. Signaling through these stimulating neural pathways produces an enhancement of inflammation attributable to the biological mechanisms of specific proinflammatory neurotransmitters activated by that specific neural circuit. As these motor circuits are stimulatory, defined by their activity to increase the output of innate immune responses, there is evidence supporting a functional approach to studying the regulation of innate immunity by combined inhibitory and excitatory neural signaling mechanisms.

A basic principle in neuroscience is that the interaction of inhibitory and excitatory neural circuits mediates the output of a neural network, and determines the final state of organ function. Major forms of information processing in the nervous system are routinely defined by the identity of the excitatory or inhibitory neurotransmitter released in response to specific action potentials. Accordingly, depending on the neurotransmitters released, neural circuits can either inhibit inflammation, as in the case of cholinergic signals, or enhance inflammation, as in the cases of proinflammatory neuropeptides, adrenergic signals, and substance $P$. The functional output depends on the neurotransmitter receptors, rather than the neurotransmitters per se, as in the case of adrenergic signaling by norepinephrine secreting neurons, which can enhance or inhibit inflammation, depending on whether the principle receptors engaged are $\alpha$ or $\beta$ adrenergic GPRs. Indeed, most innervated tissues receive input from circuits that release immune inhibitory and immune excitatory signals, so the net effect of neural control on the immune response will depend on the sum total interaction of molecular mechanisms resulting from ligand receptor signal transduction in a cell-specific and neurotransmitter-specific path. 
To this point, I have argued that the output of neural reflex circuits regulates the innate immune response to pathogens, and that these circuits can be activated by IL-1 and other endogenous products of inflammation and tissue damage. This is in keeping with pervasive immunological thinking that the innate immune system is the primary sensor of the presence of pathogens. Next, consider that the regulatory intersection between the nervous and immune systems can also originate with pathogen sensing. It is clear that innate immune system receptors respond to pathogen-associated molecular patterns during early phases of infection, and that clonal expansion of lymphocytes expressing specific pathogen sensing receptors confers long-term protection against subsequent infections. But how early is "early," and what role might the nervous system play if it detects the presence of microbes or tissue damage first, before innate immune responses? Can neurons respond to bacteria directly, or must there be an intermediate sensing step of microbial recognition by the innate immune system?

Consider that peripheral sensory neurons form a dense meshlike network that envelops and covers all tissues exposed to the external environment, including the epithelial lining of the skin and soft tissues, as well as the pulmonary, genitourinary, and gastrointestinal surfaces. Wherever microbes might gain entry, a sensory neural net is in place to respond. Specialized "nociceptor" neurons can be activated to transmit afferent action potentials by exposure to specific molecules. In C. elegans, with its limited repertoire of neurons, glycolipids containing ascarylose (3,6-dideoxy-L-arabino-hexose), termed "ascarosides," stimulate specific nocioreceptor neurons that lead to discrete behavioral responses (Ludewig and Schroeder 2013). For example, exposure of worms to ascr\#3 mediates either attraction or repulsion of hermaphrodites and males, whereas chemical modification of ascr\#3 by addition of a tryptophan adduct (producing "Icas\#3") produces entirely different aggregation and attraction behavior. In this example, discrete chemical modification of a well-characterized molecular entity provides a differential sensory input into reflex circuits that produce visibly perceptible behavioral responses.

Stimulation of nocioreceptors produces graded potentials that propagate short distances, and can lead to stimulation of action potentials in a frequency that reflects the intensity of the original stimulus. These action potentials travel longer distances along axons from the peripheral tissue into the central nervous system, where the incoming information can be relayed to the brain stem, and can activate an interneuron to stimulate a motor arc of a simple reflex that returns to the periphery. Another feature of some nocioceptive neurons is that signals propagating toward the central nervous system can be diverted at branch points to travel back toward the periphery in a phenomenon termed an "axon reflex" (Yaprak 2008). By this mechanism, TNF, IL-1, prostaglandins, substance P, and other molecular products of inflammation stimulate nocioceptive neurons to stream signals to the brain stem and back into the regional tissues. Functional expression of receptors for cytokines (e.g., IL-1R, TNFR), pathogen-associated molecules (e.g., TLR3, TLR4, TLR7, TLR9), and damage-associated molecules (e.g., RAGE, P2X3) have all been implicated in mediating neuronal hyperpolarization and signaling (Chiu et al. 2012).

From the neuroscience perspective, there is nothing particularly curious about the nervous system sensing molecular input from the milieu interior and exterior. Changes in the molecular composition of the environment directly mediate the generation of action potentials, and initiate the neural platform for responding to threat, which stimulates learning. If one approaches this from within a narrow immunology perspective, there will be significant bias in favor of theories that cells within the innate immune system, not the nervous system, are the primary sensing apparatus to detect pathogens. From an evolutionary perspective, however, there is no reason to ascribe innate sensing primacy to the immune system. Indeed, the facts argue to the contrary. Atomic structural mechanistic studies of molecular signaling through neurons in C. elegans, the evolutionary precur- 
K.J. Tracey

sors to mammalian neuronal reflexes, react differentially to subtle changes in molecular structure. It can be strongly argued that neurons, as well as innate immune cells, evolved to "sense" molecules derived from pathogens in a manner that initiates highly specific and coordinated responses that benefit survival, and that evolution would preserve these advantages.

The recent discovery by Clifford Woolf and colleagues that mammalian nocioceptors sense pathogens independently from the innate immune system indicates that some adjustment will have to be made to prior immune-centric sensing theories (Chiu et al. 2013). Inoculation of bacteria into mice produced pain and inflammatory responses, even when animals were rendered genetically deficient in TLR and MyD88dependent signal transduction mechanisms. $N$-formylated peptides derived from Gramnegative and Gram-positive bacteria are sensed by G-protein-coupled formyl peptide receptors (FPRs) on neurons. Administration of E. coliderived peptide (fMLF), and Staphylococcus aureus-derived peptide (fMIFL) to mice-activated calcium flux in nocioceptive neurons that mediate pain and mechanical hypersensitivity. Exposure of the $S$. aureus-derived poreforming toxin, $\alpha$-haemolysin, induced a concentration-dependent calcium flux in sensory neurons, which express A disintegrin and metalloprotease 10 (ADAM10), implicated in membrane pore assembly. The assembly of molecular pores in response to $\alpha$-haemolysin is sufficient to mediate ion flux and generate action potentials in sensory neurons. Mice rendered deficient in nocioceptive neurons (Nav1.8-Cre/ diphtheria toxin A mice) and exposed to $S$. aureus-developed enhanced inflammatory responses, including increased neutrophil and monocyte infiltration, increased lymph node swelling, and increased production of TNF. Thus, the interaction of pathogen-associated molecules on neurons directly activated sensory information, transmitted as action potentials, to mediate a locally inhibitory neural circuit that suppresses inflammation (Chiu et al. 2013). This direct mechanistic evidence establishes that pathogen-derived molecules can be sensed by neurons, that specific molecular products activate specific neuronal mechanisms that culminate in the generation of action potentials, that localized neural reflexes contribute to regional regulation of innate immunity, and that all of this occurs at the earliest, immediate stages of host responses that are associated with learning and, eventually, memory.

\section{CONCLUSIONS}

Viewing the onset of immunity as commencing with neural sensing independent from, and costimulatory with, immune cell sensing has profound implications to immunological thinking. For students interested in the functional activity of hematopoietic cells, it will remain an option, of course, to continue investigation into the interaction between specific microbes and pathogen-associated molecules with specific cognate receptors expressed in cells from the myeloid lineage. This can be furthered in exquisite molecular detail by pursuing signal transduction mechanisms that converge on gene expression patterns. Students interested in understanding how immunological memory develops in mammals during infection, and those interested in understanding how the innervated host operates as a physiologically regulated animal within constraints of a stable internal milieu, will take a different approach. The revolutionary new approach to immunological thinking will embrace the mechanisms of inhibitory and excitatory neural feedback loops that function from the onset of infection, and are coordinated by brain stem reflexes and regional axonal reflexes, to modulate the output of the innate immune cells that independently sense the presence of microbial agents.

Revolutions can be messy business, and predicting the outcome even messier. But sometimes, as perhaps illustrated by Janeway's prescience in defining the second revolution in immunological thinking, the outcome can be predetermined by facts. At present, nearly 25 years after Janeway's prediction, innate immunity has been elevated to heightened preeminence in immunological thinking. There is an intense and appropriate focus on understanding the role of innate immunity in the ac- 
quisition of adaptive immunity. Looking to the coming revolution, there is clear evidence today that innate immunity is regulated by neural reflexes activated by pathogens and host-derived mediators of infection, inflammation, and injury; and there is strongly suggestive evidence that neural signals modulate adaptive immunity as well (Wong et al. 2011; Arima et al. 2012; MinaOsorio et al. 2012). From my vantage point, this has all the makings of a revolution in immunological thinking that is under way owing to a convergence, not a clashing, of two major fields. A complete understanding of immunity requires fully understanding the neural response to infection and injury (Andersson and Tracey 2012b). Tools provided by molecular biology, neurophysiology, and cell biology render this a particularly exciting time to embrace this intersection of neuroscience and immunology, and to consider that advances from worms to mammals hold promise for advances in the clinic.

\section{REFERENCES}

Aballay A. 2013. Role of the nervous system in the control of proteostasis during innate immune activation: Insights from C. elegans (ed. Goldman WE) PLoS Pathog 9: e1003433.

Andersson U, Tracey KJ. 2012a. Neural reflexes in inflammation and immunity. J Exp Med 209: 1057-1068.

Andersson U, Tracey KJ. 2012b. Reflex principles of immunological homeostasis. Annu Rev Immunol 30: 313-335.

Arima Y, Harada M, Kamimura D, Park J-H, Kawano F, Yull FE, Kawamoto T, Iwakura Y, Betz U, Márquez G, et al. 2012. Regional neural activation defines a gateway for autoreactive $\mathrm{T}$ cells to cross the blood-brain barrier. Cell 148: 447-457.

Borovikova LV, Ivanova S, Zhang M, Yang H, Botchkina GI, Watkins LR, Wang H, Abumrad N, Eaton JW, Tracey KJ. 2000. Vagus nerve stimulation attenuates the systemic inflammatory response to endotoxin. Nature 405: 458 462.

Chiu IM, Von Hehn CA, Woolf CJ. 2012. Neurogenic inflammation and the peripheral nervous system in host defense and immunopathology. Nat Neurosci 15: $1063-$ 1067.

Chiu IM, Heesters B, Ghasemlou N, Von Hehn C, Zhao F, Tran J, Wainger B, Strominger A, Muralidharan S, Horswill AR, et al. 2013. Bacteria activate sensory neurons that modulate pain and inflammation. Nature 501: $52-57$.

De Jonge WJ, van der Zanden EP, The FO, Bijlsma MF, van Westerloo DJ, Bennink RJ, Berthoud H-R, Uematsu S, Akira S, van den Wijngaard RM, et al. 2005. Stimulation of the vagus nerve attenuates macrophage activation by activating the Jak2-STAT3 signaling pathway. Nat Immunol 6: 844-851.

Dubos R. 1998. So human an animal: How we are shaped by surroundings and events. Transaction, Piscataway, NJ.

Fairchild KD, Srinivasan V, Moorman JR, Gaykema RP, Goehler LE. 2011. Pathogen-induced heart rate changes associated with cholinergic nervous system activation. Am J Physiol Regul Integr Comp Physiol 300: R330-R339.

Huston JM, Ochani M, Rosas-Ballina M, Liao H, Ochani K, Pavlov VA, Gallowitsch-Puerta M, Ashok M, Czura CJ, Foxwell B, et al. 2006. Splenectomy inactivates the cholinergic antiinflammatory pathway during lethal endotoxemia and polymicrobial sepsis. J Exp Med 203: $1623-1628$.

Janeway C. 1989. Approaching the asymptote? Evolution and revolution in immunology. Cold Spring Harb Symp Quant Biol 54: 1-13.

Lu B, Kwan K, Levine YA, Olofsson PS, Yang H, Li J, Joshi S, Wang H, Andersson U, Chavan SS, Tracey KJ. 2014. $\alpha 7$ nicotinic acetylcholine receptor signaling inhibits inflammasome activation by preventing mitochondrial DNA release. Mol Med doi: 10.2119/molmed.2013. 00117.

Ludewig AH and Schroeder FC. 2013. Ascaroside signaling in C. elegans. In WormBook (ed. The C. elegans Research Community), doi/10.1895/wormbook.1.155.1.

Mina-Osorio P, Rosas-Ballina M, Valdes-Ferrer SI, Al-Abed Y, Tracey KJ, Diamond B. 2012. Neural signaling in the spleen controls B-cell responses to blood-borne antigen. Mol Med 18: 618-627.

Niijima A. 1996. The afferent discharges from sensors for interleukin $1 \beta$ in the hepatoportal system in the anesthetized rat. J Auton Nerv Syst 61: 287-291.

Niijima A, Hori T, Aou S, Oomura Y. 1991. The effects of interleukin- $1 \beta$ on the activity of adrenal, splenic and renal sympathetic nerves in the rat. J Auton Nerv Syst 36: 183-192.

Pradel E, Zhang Y, Pujol N, Matsuyama T, Bargmann CI, Ewbank JJ. 2007. Detection and avoidance of a natural product from the pathogenic bacterium Serratia marcescens by Caenorhabditis elegans. Proc Natl Acad Sci 104: 2295-300.

Rosas-Ballina M, Ochani M, Parrish WR, Ochani K, Harris YT, Huston JM, Chavan S, Tracey KJ. 2008. Splenic nerve is required for cholinergic antiinflammatory pathway control of TNF in endotoxemia. Proc Natl Acad Sci 105: 11008-11013.

Rosas-Ballina M, Olofsson PS, Ochani M, Valdés-Ferrer SI, Levine Y, Reardon C, Tusche MW, Pavlov VA, Andersson U, Chavan S, et al. 2011. Acetylcholine-synthesizing T cells relay neural signals in a vagus nerve circuit. Science 334: $98-101$.

Sherrington SCS. 1906. The integrative action of the nervous system. Yale University Press, New Haven.

Styer KL, Singh V, Macosko E, Steele SE, Bargmann CI, Aballay A. 2008. Innate immunity in Caenorhabditis elegans is regulated by neurons expressing NPR-1/GPCR. Science 322: 460-464.

Sun J, Singh V, Kajino-Sakamoto R, Aballay A. 2011. Neuronal GPCR controls innate immunity by regulating non- 
K.J. Tracey

canonical unfolded protein response genes. Science 332: $729-732$.

Tracey KJ. 2002. The inflammatory reflex. Nature 420: $853-$ 859.

Tracey KJ. 2007. Physiology and immunology of the cholinergic antiinflammatory pathway. J Clin Invest 117: 289296.

Tracey KJ. 2009. Reflex control of immunity. Nat Rev Immunol 9: 418-428.

Tracey KJ, Beutler B, Lowry SF, Merryweather J, Wolpe S, Milsark IW, Hariri RJ, Fahey TJ, Zentella A, Albert JD. 1986. Shock and tissue injury induced by recombinant human cachectin. Science 234: 470-474.

Tracey KJ, Fong Y, Hesse DG, Manogue KR, Lee AT, Kuo GC, Lowry SF, Cerami A. 1987. Anti-cachectin/TNF monoclonal antibodies prevent septic shock during lethal bacteraemia. Nature 330: 662-664.
Wang HH, Yu M, Ochani M, Amella CA, Tanovic M, Susarla S, Li JH, Yang H, Ulloa L, Al-Abed Y, et al. 2003. Nicotinic acetylcholine receptor $\alpha 7$ subunit is an essential regulator of inflammation. Nature 421: 384-388.

Wang H, Liao H, Ochani M, Justiniani M, Lin X, Hong L, Alabed Y, Wang H, Metz C, Miller EJ, et al. 2004. Cholinergic agonists inhibit HMGB1 release and improve survival in experimental sepsis. Nat Med 10: 1216-1221.

Watkins L, Goehler L, Relton J. 1995. Blockade of interleukin-1 induced hyperthermia by subdiaphragmatic vagotomy: Evidence for vagal mediation of immune-brain communication. Neuroscience 183: 27-31.

Wong CHY, Jenne CN, Lee W-Y, Léger C, Kubes P. 2011. Functional innervation of hepatic iNKT cells is immunosuppressive following stroke. Science 334: 101-105.

Yaprak M. 2008. The axon reflex. Neuroanatomy 7: 17-19. 


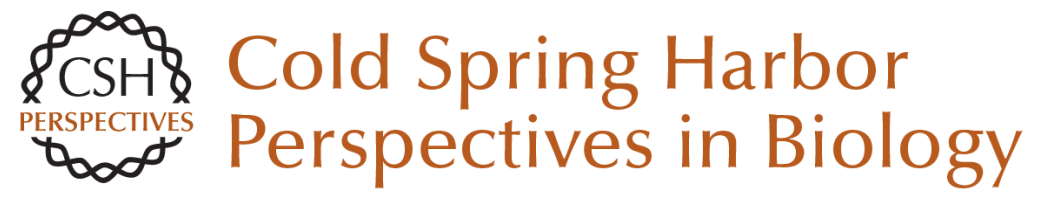

\section{Approaching the Next Revolution? Evolutionary Integration of Neural and Immune Pathogen Sensing and Response}

Kevin J. Tracey

Cold Spring Harb Perspect Biol 2015; doi: 10.1101/cshperspect.a016360 originally published online November 6, 2014

Subject Collection Innate Immunity and Inflammation

Group 2 Innate Lymphoid Cells in Health and

Disease

Brian S. Kim and David Artis

Inflammation and the Blood Microvascular

System

Jordan S. Pober and William C. Sessa

Sinusoidal Immunity: Macrophages at the

Lymphohematopoietic Interface

Siamon Gordon, Annette Plüddemann and Subhankar Mukhopadhyay

Allergic Inflammation--Innately Homeostatic Laurence E. Cheng and Richard M. Locksley

Approaching the Next Revolution? Evolutionary Integration of Neural and Immune Pathogen

Sensing and Response

Kevin J. Tracey

Inflammasomes

Marcel R. de Zoete, Noah W. Palm, Shu Zhu, et al.

IL-6 in Inflammation, Immunity, and Disease Toshio Tanaka, Masashi Narazaki and Tadamitsu Kishimoto
The Chemokine System in Innate Immunity

Caroline L. Sokol and Andrew D. Luster

Microbial Sensing by Toll-Like Receptors and

Intracellular Nucleic Acid Sensors

Surya Pandey, Taro Kawai and Shizuo Akira

Tumor Necrosis Factor Superfamily in Innate Immunity and Inflammation John Sedý, Vasileios Bekiaris and Carl F. Ware

Emerging Principles Governing Signal

Transduction by Pattern-Recognition Receptors Jonathan C. Kagan and Gregory M. Barton

Lipid Mediators in the Resolution of Inflammation Charles N. Serhan, Nan Chiang, Jesmond Dalli, et al.

Transcriptional Control of Inflammatory

Responses

Stephen T. Smale and Gioacchino Natoli

DNA Degradation and Its Defects

Kohki Kawane, Kou Motani and Shigekazu Nagata

For additional articles in this collection, see http://cshperspectives.cshlp.org/cgi/collection/

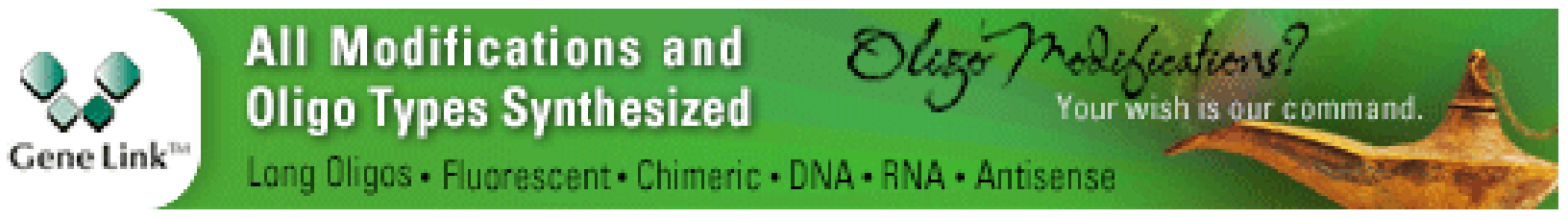

Copyright @ 2015 Cold Spring Harbor Laboratory Press; all rights reserved 